Research paper

\title{
Structure-based design of novel quinoxaline-2-carboxylic acids and analogues as Pim-1 inhibitors
}

\author{
Bruno Oyallon ${ }^{a}$, Marie Brachet-Botineau ${ }^{\text {b, c }}$, Cédric Logé ${ }^{\mathrm{d}}$, Pascal Bonnet ${ }^{\mathrm{e}}$, \\ Mohamed Souab ${ }^{\mathrm{f}}$, Thomas Robert ${ }^{\mathrm{f}}$, Sandrine Ruchaud ${ }^{\mathrm{f}}$, Stéphane Bach ${ }^{\mathrm{f}}$, \\ Pascal Berthelot ${ }^{g}$, Fabrice Gouilleux ${ }^{\mathrm{b}}$, Marie-Claude Viaud-Massuard ${ }^{\mathrm{a}}$, \\ Caroline Denevault-Sabourin ${ }^{\text {a, * }}$

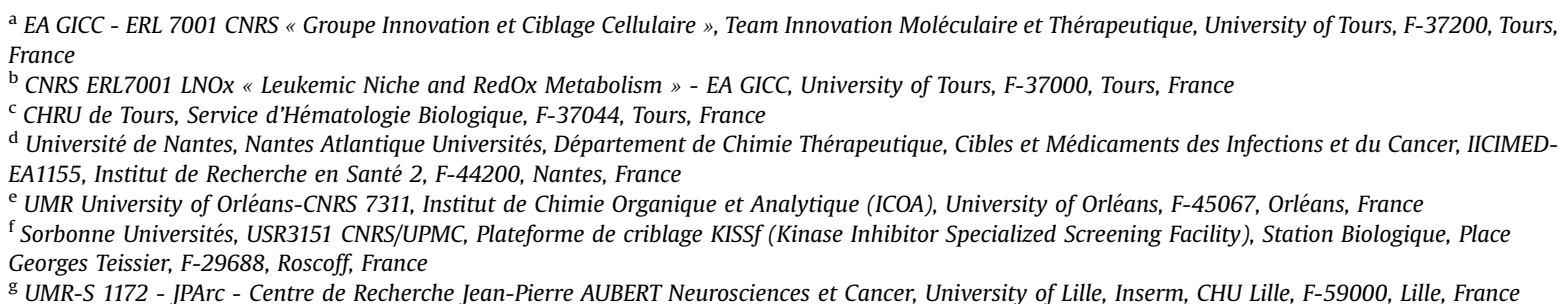

\section{A R T I C L E I N F O}

\section{Article history:}

Received 27 February 2018

Received in revised form 20 April 2018

Accepted 28 April 2018

Available online 11 May 2018

\section{Keywords:}

Quinoxaline

Pim-1

Kinase inhibitor

Anticancer targeted therapy

\begin{abstract}
A B S T R A C T
We identified a new series of quinoxaline-2-carboxylic acid derivatives, targeting the human proviral integration site for Moloney murine leukemia virus-1 (HsPim-1) kinase. Seventeen analogues were synthesized providing useful insight into structure-activity relationships studied. Docking studies realized in the ATP pocket of $H s$ Pim-1 are consistent with an unclassical binding mode of these inhibitors. The lead compound $\mathbf{1}$ was able to block HsPim-1 enzymatic activity at nanomolar concentrations ( IC $_{50}$ of $74 \mathrm{nM}$ ), with a good selectivity profile against a panel of mammalian protein kinases. In vitro studies on the human chronic myeloid leukemia cell line KU812 showed an antitumor activity at micromolar concentrations. As a result, compound 1 represents a promising lead for the design of novel anticancer targeted therapies.
\end{abstract}

() 2018 Elsevier Masson SAS. All rights reserved.

\section{Introduction}

Proviral integration site for Moloney murine leukemia virus (Pim) kinases belong to a small family of constitutively activated proto-oncogenic serine/threonine protein kinases, constituted of

Abbreviations: IC50, 50\% inhibitory concentration; SAR, structure-activity relationships; Pim, proviral integration site of Moloney murine leukemia virus; CML, chronic myeloid leukemia; DYRK1A, dual specificity tyrosine phosphorylation regulated kinase $1 \mathrm{~A}$; $\mathrm{CDK}$, cyclin-dependent kinase; Haspin, haploid germ cellspecific nuclear protein kinase; CLK1, CDC2-like kinase 1; CK1, casein kinase 1; GSK3, glycogen synthase kinase 3.

* Corresponding author. Department of Innovation Moléculaire et Thérapeutique EA GICC - ERL 7001 CNRS, University of Tours, 31 avenue Monge, F-37200, Tours, France.

E-mail address: caroline.sabourin@univ-tours.fr (C. Denevault-Sabourin). three isoforms: Pim-1, Pim-2 and Pim-3 [1]. These oncoproteins control many cellular functions like cell cycle regulation, apoptosis, cell survival, proliferation and differentiation [2,3], and are overexpressed in a large number of human cancer types, such as hematopoietic malignancies [4,5] and solid cancers (e. g. bladder [6], prostate [7], breast [8] or oral cancers [9]). These kinases are positive regulators of cell cycle progression at G1/S and G2/M checkpoints, and inhibit apoptosis, acting as oncogenic survival factors [10]. Interestingly, it has been demonstrated that Pim $1^{-1-} 2^{-1-} 3^{-1-}$ triple knockout mice were viable and fertile, which make these kinases very interesting for targeted cancer therapies [11].

Recently, Pim-1 has been shown to play a significant role in cancer stem cells growth, and in resistance to chemotherapy drugs, promoting multiple drug resistance [12,13]. This kinase is thus considered as a relevant target for cancer therapy and a large variety of small molecule inhibitors have been developed [14-18]. 
Many of these Pim-1 kinase inhibitors demonstrated significant in vitro activity in cancer cell lines and in different in vivo tumor xenograft models, and clinical trials are currently ongoing for the most promising candidates [14,18].

A remarkable characteristic of Pim-1 active site in comparison to other protein kinases is the presence of an original hinge region (region containing backbone peptide atoms that forms hydrogen bond interactions (H-bonds) with the adenine moiety of ATP). Indeed, this region contains a proline residue (Pro123), which has no $\mathrm{H}$-bond donor property and precludes the formation of one of the conserved $\mathrm{H}$-bond involving the hinge backbone and the ATP adenine ring, as it can be observed in other kinases. Thus, Pim-1 bounds ATP via only one hinge H-bond between the ATP adenine amino moiety and the backbone carbonyl of glutamate 121 (Glu121). Moreover, the insertion in the hinge of a valine (Val126), absent in other kinases, changes the hinge conformation, enlarging the catalytic pocket. This unique feature can be exploited for the design of selective inhibitors [19].

The vast majority of Pim-1 inhibitors mainly act as ATP competitive inhibitors, targeting the ATP-binding pocket. They can be classified into two categories: ATP-mimetics, which bind to the Glu121 residue of the hinge region, and non-ATP mimetics, which interact with the ATP binding cleft in a different manner from ATP [20].

In a continuing effort to develop new small molecule inhibitors with anticancer properties, our laboratory has been recently focusing on the study of new inhibitors of the signal transducer and activator of transcription 5 (STAT5) activation and expression and their interest in chronic myeloid leukemia (CML) [21]. Indeed, the STAT family transcription factors are commonly activated in cancer by upstream mutations or cell surface signaling molecules. It has been demonstrated that the Pim kinases are induced by the STAT family transcription factors (particularly STAT 3/5) [14]. Regarding the potential of Pim-1 as target in cancer therapy and particularly in leukemia [22,23], we decided to further explore the STAT signaling pathway, by developing new Pim-1 kinase specific inhibitors. In this purpose, we first performed a target-based approach, by realizing a focused in vitro screening of our chemical library on a limited panel of kinases, comprising Homo sapiens Pim-1 (HsPim1 ), allowing the identification of the quinoxaline-2-carboxylic acid $\mathbf{1}$ as a new lead compound (Fig. 1). This molecule was able to inhibit the in vitro enzymatic activity of $\mathrm{HsPim}-1$ with an $\mathrm{IC}_{50}$ of $74 \mathrm{nM}$.

Docking studies, using program GOLD (GOLD version 4.0; CCDC, Cambridge, UK), were performed to understand the binding interactions between the lead compound $\mathbf{1}$ and the ATP pocket of HsPim-1 (PDB ID 3A99) (Fig. 2). Data analysis suggests that the carboxylate group of this molecule can form a key salt bridge with the protonated amino group side chain of catalytic Lys67, as it has already been described in other Pim-1 inhibitors [24,25], and shares also a H-bond interaction with the backbone NH of Asp186 belonging to the DFG motif. Additionally, an H-bond interaction between the 3-hydroxyphenyl moiety and the carboxylate group of

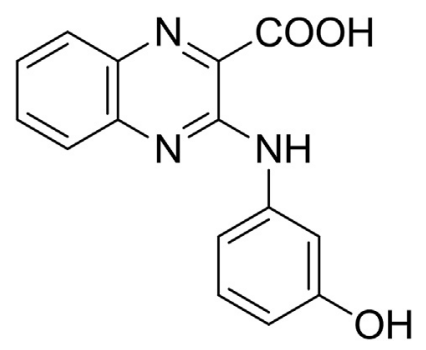

Fig. 1. Chemical structure of compound $\mathbf{1}$. residue Asp186 can be observed. These studies suggested that compound 1 could act as an ATP competitive inhibitor, with a nonATP mimetic binding mode.

Sixteen new analogues were then synthesized, exploiting the unique sequence of $\mathrm{HsPim}-1$ ATP-binding cleft.

We report herein the design, synthesis, structure-activity relationships (SAR) and in vitro evaluations of this new class of Pim-1 inhibitors.

\section{Chemistry}

The preparation of quinoxaline-2-carboxylic acids $\mathbf{1}, \mathbf{5 c}$-e, and $\mathbf{5 h}-\mathbf{i}$ and potassium carboxylate salts $\mathbf{5 b}$, and $\mathbf{5 g}$ was performed as shown in Scheme 1 by amination of the intermediate ethyl 3chloroquinoxaline-2-carboxylate $\mathbf{3}$ with the appropriate amine derivatives.

The synthesis of ethyl 3-chloroquinoxaline-2-carboxylate 3 was achieved in two steps from commercial $o$-phenylenediamine according to literature procedures [26,27] (Scheme 1). First, the ophenylenediamine was condensed with diethyl 2-oxomalonate in the presence of citric acid ( $3 \mathrm{~mol} \%$ ) at room temperature in ethanol to give ester 2, which was then chlorinated using $\mathrm{N}, \mathrm{N}$-dimethylformamide (DMF) as a catalyst in refluxing phosphorous oxychloride, affording the intermediate $\mathbf{3}$ in quantitative yield.

Access to quinoxaline-2-carboxylic acids, and potassium carboxylate salts was then performed using a two-step synthetic pathway. Thus, intermediate $\mathbf{3}$ undergoes initial nucleophilic aromatic substitution with the appropriate amine in presence of $p$-TSA as a catalyst in refluxing absolute ethanol to give esters $\mathbf{4 a - f}$ and 4h-i [28]. For compound 4f, a supplementary step of tert-butyloxycarbonyl (Boc) group deprotection, using trifluoroacetic acid in dichloromethane (DCM), was necessary to obtain the derivative $\mathbf{4 g}$. Then, hydrolysis of the intermediate ethyl esters $\mathbf{4 a - c}, \mathbf{4 e}$, and $\mathbf{4 g - i}$ with potassium carbonate in refluxing $80 \%$ aqueous methanol was performed. The potassium salts $\mathbf{5 b}$ and $\mathbf{5 g}$ were thus obtained without any treatment. A subsequent acidification with a citric acid aqueous solution was realized to afford acids $\mathbf{1}, \mathbf{5 c}-\mathbf{e}$, and $\mathbf{5 h - i}$.

Ester 4d was saponified using a 10\% aqueous sodium hydroxide solution in refluxing ethanol, leading, after acidification with a citric acid aqueous solution, to the corresponding carboxylic acid $5 d$.

Finally, synthesis of the carboxamide $\mathbf{6}$ was achieved from acid 5c, using $N$-methylmorpholine and ethyl chloroformate in dichloromethane at $0{ }^{\circ} \mathrm{C}$, followed by the addition of a $28 \%$ ammonium hydroxide solution.

\section{Results and discussion}

\subsection{Enzymatic assays}

\subsubsection{Pim-1 enzymatic activity inhibition}

Compounds were first evaluated for their efficacy to inhibit the in vitro enzymatic activity of $\mathrm{HsPim}-1$, using a luminescence-based kinase assay [29]. Compounds that displayed HsPim-1 IC $50>10 \mu \mathrm{M}$ were considered inactive.

To get closer insight into the potential binding mode of our compounds within the ATP binding pocket of HsPim-1, we first decided to structurally vary the substitution patterns of the quinoxaline scaffold of $\mathbf{1}$ in position 3 (Table 1). Docking analysis revealed that the hydroxyl moiety of the phenyl ring in this position could be able to form an $\mathrm{H}$-bond interaction with the carboxylate side chain of residue Asp186 of the HsPim-1 ATP binding pocket. Interestingly, it appears that position of the hydroxyl group on the phenyl ring strongly modulates compound activity, since modification from meta $(\mathbf{1})$ to para $(\mathbf{5 e})$ or ortho $(\mathbf{5 i})$ position reduced 


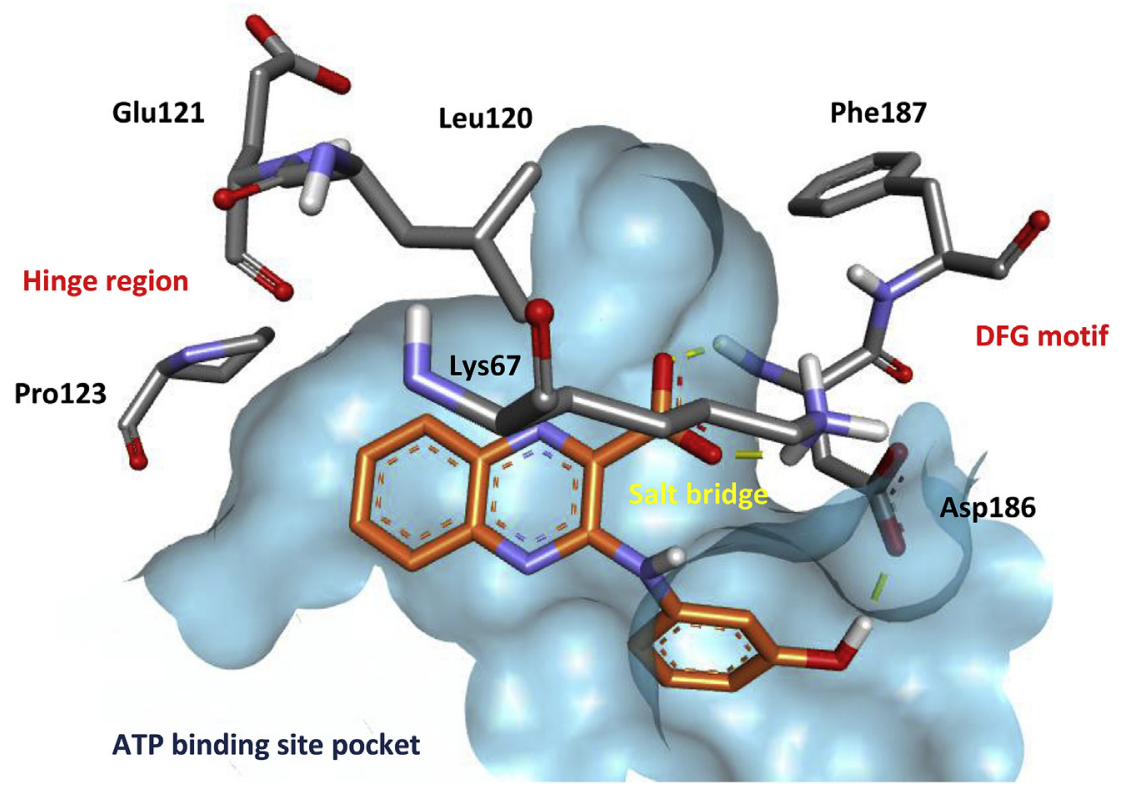

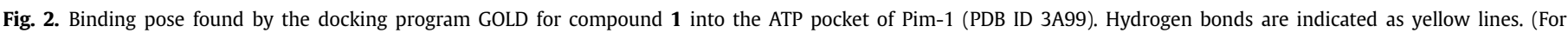
interpretation of the references to colour in this figure legend, the reader is referred to the Web version of this article.)
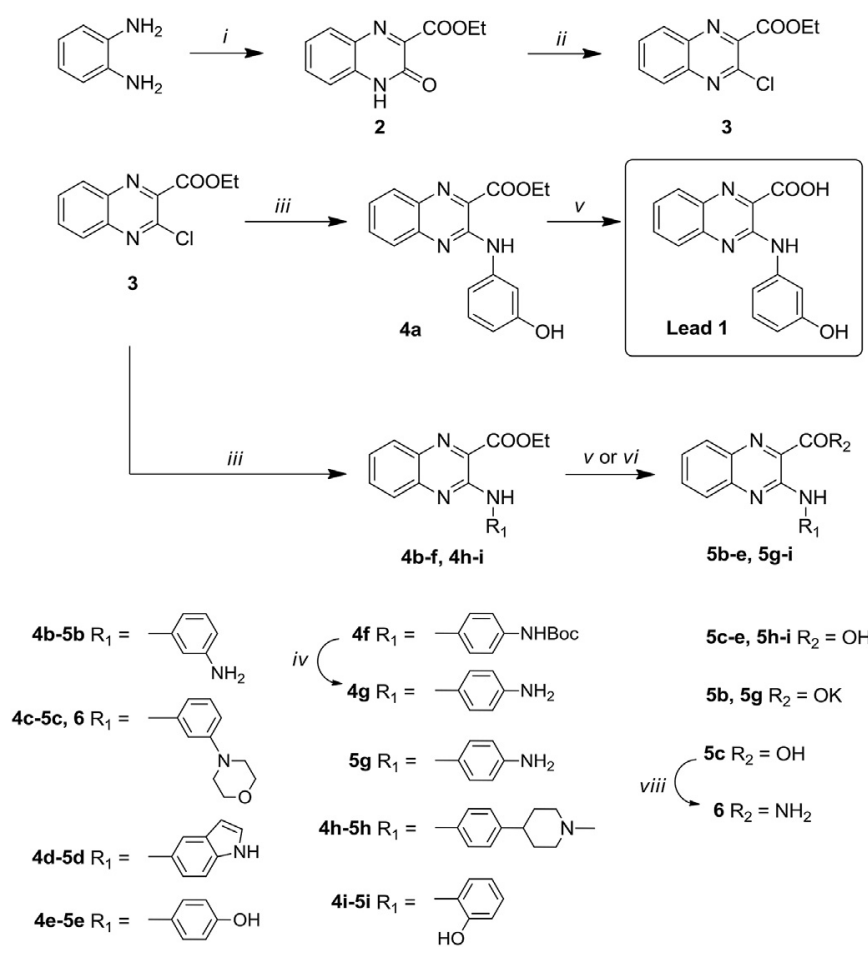

Scheme 1. Reagents and conditions: (i) diethyl 2-oxomalonate (1 eq), citric acid (3 mol \%), EtOH, rt, $10 \mathrm{~min}, 79 \%$; (ii) DMF (cat.), $\mathrm{POCl}_{3}, 0{ }^{\circ} \mathrm{C}$, and then reflux, $30 \mathrm{~min}, 100 \%$; (iii) amine (1.1-3 eq), p-toluenesulfonic acid (cat.), EtOH, reflux, 20-112 h, 32-93\%; (iv) TFA (20\%), DCM, rt, 6 h, 96\%; (v) $\mathrm{K}_{2} \mathrm{CO}_{3}$ (1-4 eq), MeOH/ $\mathrm{H}_{2} \mathrm{O}$ (4/1), reflux, 4 h, 49-100\%; (vi) $\mathrm{NaOH}$ (10\%), EtOH, reflux, 18 h, 41\%; (vii) ClCOOEt (1.5 eq), NMM (2 eq), DCM, $0{ }^{\circ} \mathrm{C}$ $1 \mathrm{~h}$, and $\mathrm{NH}_{4} \mathrm{OH}$, rt, overnight, $100 \%$.

significantly the inhibitory potency (Table 1 , entries 2,11 , and 17). Thus, compounds $5 \mathbf{e}$ and $\mathbf{5 i}$ maintained a submicromolar activity on $\mathrm{HsPim}-1$ ( IC $_{50}$ of $0.29 \mu \mathrm{M}$ and $0.76 \mu \mathrm{M}$, respectively) but were less potent (4-fold, 10-fold, respectively) than lead compound 1 $\left(\mathrm{IC}_{50}\right.$ of $\left.74 \mathrm{nM}\right)$.
Surprisingly, the replacement of the hydroxyl moiety of compound 1 by an amino group, able to form an H-bond with Asp186, led to a significant loss of potency $\left(\mathbf{5 b}, \mathrm{IC}_{50}\right.$ of $2.80 \mu \mathrm{M}, 38$-fold lower). However, as expected, the substitution by a morpholino group, suppressing the formation of an $\mathrm{H}$-bond, was not favorable for the activity, as shown by derivative $5 \mathrm{c}\left(\mathrm{IC}_{50}\right.$ of $\left.1.01 \mu \mathrm{M}\right)$. Again, the para substitution on the phenyl ring was deleterious for the activity, as shown by derivative $\mathbf{5 g}$ which was $>3.5$-fold less active than its "meta" analogue $\mathbf{5 b}$, and by the inactive compound $\mathbf{5 h}$ (Table 1, entries 4,13 and 15).

Finally, replacement of the 3-hydroxyphenyl moiety of compound $\mathbf{1}$ by an $1 \mathrm{H}$-indol-5-yl group (5d) led to a drastic loss of potency (Table 1, entry 9).

Docking analysis also suggested that the carboxylate function in position 2 of the quinoxaline scaffold of compound $\mathbf{1}$ was crucial for the activity, establishing, notably, a key salt bridge with the catalytic Lys67. However, carboxylic acids are known to be responsible for limited permeability across biological membranes, metabolic instability, and potential adverse effects [30]. To circumvent these issues, and to confirm the results of the modeling studies, we evaluated ethyl ester derivatives (4a-e and $\mathbf{4 g}-\mathbf{i}$ ) of all synthesized acids and carboxylate salts, and we replaced the acid group of compound $\mathbf{5 c}$ by a carboxamide isosteric moiety $(\mathbf{6})$. Both ester and carboxamide functions are not able to form a salt bridge like the carboxylic acid group, resulting in a complete loss of HsPim-1 enzymatic activity inhibition (Table 1, entries 1, 3, 5, 7, 8, 10, 12, 14 and 16), highlighting the highly critical role of this type of interaction in position 2 of the quinoxaline ring.

\subsubsection{Selectivity over a panel of mammalian protein kinases}

A selectivity profile of the most active $\mathrm{HsPim}-1$ inhibitors was performed. In that purpose, most promising candidates were further evaluated in an expanded panel of mammalian protein kinases such as RnDYRK1A, HsCDK2/CyclinA, HsCDK9/CyclinT, $H s$ Haspin, MmCLK1, SscCK1 $\delta / \varepsilon$ and SscGSK3 $\alpha / \beta$. Inhibition values were determined using a luminescence-based kinase assay [29].

Similar inhibition trends were observed with 6 of the mammalian kinases tested (HsCDK2/CyclinA, HsCDK9/CyclinT, $H s$ Haspin, MmCLK1, SscCK1 $\delta / \varepsilon$ and SscGSK3 $\alpha / \beta)$, with $\mathrm{IC}_{50}>10 \mu \mathrm{M}$ 
Table 1

Enzymatic assays on $\mathrm{HsPim}-1$.

$$
\text { - }
$$

\begin{tabular}{|c|c|c|c|c|}
\hline Entry & Compd & $R_{1}$ & $R_{2}$ & HsPim-1 IC $50(\mu \mathrm{M})^{\mathrm{a}}$ \\
\hline 1 & $4 a$ & & OEt & $>10$ \\
\hline 2 & 1 & & $\mathrm{OH}$ & 0.074 \\
\hline 3 & $4 b$ & & OEt & $>10$ \\
\hline 4 & $\mathbf{5 b}$ & & $\mathrm{OK}$ & 2.80 \\
\hline 5 & $4 c$ & & OEt & $>10$ \\
\hline 6 & $5 c$ & & $\mathrm{OH}$ & 1.01 \\
\hline 7 & 6 & & $\mathrm{NH}_{2}$ & $>10$ \\
\hline 8 & 4d & & OEt & $>10$ \\
\hline 9 & $5 d$ & & $\mathrm{OH}$ & $>10$ \\
\hline 10 & $4 e$ & & OEt & $>10$ \\
\hline 11 & $5 e$ & & $\mathrm{OH}$ & 0.29 \\
\hline 12 & $4 g$ & & OEt & $>10$ \\
\hline 13 & $5 g$ & & OK & $>10$ \\
\hline 14 & $4 h$ & & OEt & $>10$ \\
\hline 15 & $5 h$ & & $\mathrm{OH}$ & $>10$ \\
\hline 16 & $4 i$ & & OEt & $>10$ \\
\hline 17 & $5 \mathbf{i}$ & & $\mathrm{OH}$ & 0.76 \\
\hline 18 & \multicolumn{3}{|c|}{ Staurosporine } & 0.031 \\
\hline
\end{tabular}

${ }^{a}$ Values are a mean of $n \geq 3$ independent experiments. Hs: Homo sapiens.

in every case, for each compound evaluated, suggesting an interesting selectivity profile against these potential off-target kinases (Table 2). Notably, we observed $>130$-fold differences between $\mathrm{IC}_{50}$ values for $H s$ Pim-1 over these mammalian kinases for our lead inhibitor 1.
In contrast, quinoxalines $\mathbf{1}, \mathbf{5 b}, \mathbf{5 e}$ and $\mathbf{5 i}$ displayed a micromolar to submicromolar inhibition of RnDYRK1A (Table 2, entries 1, 2, 4 and 5). Lead 1 exhibited nevertheless an $\mathrm{IC}_{50}$ value at least 3.5-fold higher for RnDYRK1A than for HsPim-1. Interestingly, compound $\mathbf{5 c}$, despite a less potent activity profile against HsPim-1, was more than 10 -fold selective with respect to this kinase (Table 2, entry 3 ).

\subsection{In vitro cell-based assays}

Most active HsPim-1 inhibitors were then tested in vitro on the human CML cell line KU812, overexpressing Pim-1. Cytotoxic effects were evaluated using a MTT assay, and living cells were also counted with the trypan blue dye exclusion method.

As expected, a same trend was observed between HsPim-1 enzymatic activity inhibition and in vitro cytotoxic potency. Indeed, quinoxalines with a good level of activity on $\mathrm{HsPim}$ (IC $_{50}$ of $0.074-2.80 \mu \mathrm{M})$ also exhibited in vitro cytotoxic effects on KU812 cell line with $\mathrm{EC}_{50}$ values ranging from $38.9 \pm 3.4 \mu \mathrm{M}$ to $177.5 \pm 13.1 \mu \mathrm{M}$ (Table 2). Moreover, the best HsPim-1 inhibitor 1 $\left(\mathrm{IC}_{50}\right.$ of $\left.74 \mathrm{nM}\right)$, was also the most cytotoxic compound $\left(\mathrm{EC}_{50}\right.$ of $38.9 \pm 3.4 \mu \mathrm{M})$.

\section{Conclusion}

In this study, we identified a new series of quinoxaline-2carboxylic acids and analogues, exhibiting a potent activity against the HsPim-1 oncoprotein. Among the 17 compounds synthesized, 5 significantly blocked $H s$ Pim- 1 with $\mathrm{IC}_{50}$ values in the submicromolar to low micromolar range. In particular, lead compound 1 showed the best inhibitory effect against $H s$ Pim-1, with an $\mathrm{IC}_{50}$ value of $74 \mathrm{nM}$. SAR in positions 2 and 3 of the quinoxaline scaffold confirmed the molecular modeling studies, highlighting the crucial role of the carboxylic acid function in position 2 for the HsPim-1 inhibitory activity of these compounds. In vitro studies of the 5 most potent inhibitors on the human CML cell line KU812, confirmed their interest, with antitumor activities at micromolar concentrations.

This series of compounds, and particularly lead 1, could therefore represent new attractive drug candidates for extending further pharmacomodulation studies in a way to improve their potency and selectivity profile.

\section{Experimental section}

\subsection{General remarks}

All solvents were anhydrous reagents from commercial sources. Unless otherwise noted, all chemicals and reagents were obtained commercially and used without purification. Microwave heating was carried out with a single-mode Initiator Alstra (Biotage) unit. Melting points (Mp) were determined on a Stuart capillary apparatus and are uncorrected. High-resolution mass spectra (HRMS) were performed in positive mode with an ESI source on a Q-TOF mass spectrometer (Bruker maXis) with an accuracy tolerance of $2 \mathrm{ppm}$. NMR spectra were recorded at $300 \mathrm{MHz}\left({ }^{1} \mathrm{H}\right)$ or $75 \mathrm{MHz}$ $\left({ }^{13} \mathrm{C}\right)$ on a Bruker Avance $(300 \mathrm{MHz})$ spectrometer. The chemical shifts are reported in parts per million (ppm, $\delta$ ) relative to residual deuterated solvent peaks. The abbreviations $s=$ singlet, $\mathrm{d}$ = doublet, $\mathrm{t}$ = triplet, $\mathrm{q}$ = quadruplet, $\mathrm{m}=$ multiplet and bs = broad signal were used throughout. Known compounds were prepared according to literature procedures: tert-butyl (3aminophenyl)carbamate, and tert-butyl (4-aminophenyl)carbamate [31], 4-(1-methylpiperidin-4-yl)aniline [32]. 
Table 2

Kinase selectivity profile and cell-based assays of most active quinoxalines.

\begin{tabular}{|c|c|c|c|c|c|c|c|c|c|c|c|c|}
\hline \multirow[t]{2}{*}{ Entry } & \multirow[t]{2}{*}{ Cpd } & \multirow[t]{2}{*}{$R_{1}$} & \multirow[t]{2}{*}{$R_{2}$} & \multicolumn{8}{|c|}{ Kinase enzymatic $\mathrm{IC}_{50}(\mu \mathrm{M})^{\mathrm{a}}$} & \multirow{2}{*}{$\frac{\mathrm{EC}_{50}(\mu \mathrm{M})^{\mathrm{a}}}{\mathrm{KU} 812^{\mathrm{b}}}$} \\
\hline & & & & HsPim-1 & RnDYRK1A & HsCDK2/CyclinA & HsCDK9/CyclinT & Hs Haspin & $M m$ CLK1 & Ssc CK $1 \delta / \varepsilon$ & Ssc GSK3 $\alpha / \beta$ & \\
\hline 1 & 1 & - & $\mathrm{H}$ & 0.074 & 0.27 & $>10$ & $>10$ & $>10$ & $>10$ & $>10$ & $>10$ & $38.9 \pm 3.4$ \\
\hline 2 & $\mathbf{5 b}$ & & $\mathrm{K}$ & 2.80 & 1.67 & $>10$ & $>10$ & $>10$ & $>10$ & $>10$ & $>10$ & $63.8 \pm 1.9$ \\
\hline 3 & $5 c$ & & $\mathrm{H}$ & 1.01 & $>10$ & $>10$ & $>10$ & $>10$ & $>10$ & $>10$ & $>10$ & $57.3 \pm 6.1$ \\
\hline 4 & $5 e$ & & $\mathrm{H}$ & 0.29 & 0.098 & $>10$ & $>10$ & $>10$ & $>10$ & $>10$ & $>10$ & $41.7 \pm 3.7$ \\
\hline 5 & $5 \mathbf{i}$ & & $\mathrm{H}$ & 0.76 & 0.74 & $>10$ & $>10$ & $>10$ & $>10$ & $>10$ & $>10$ & $177.5 \pm 13.1$ \\
\hline 6 & \multicolumn{3}{|c|}{ Imatinib mesylate } & ND & ND & ND & ND & ND & ND & ND & ND & $0.6 \pm 0.02$ \\
\hline
\end{tabular}

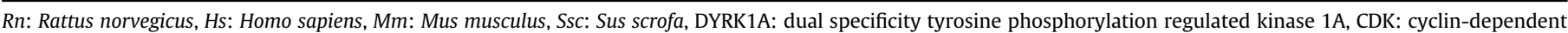
kinase, Haspin: haploid germ cell-specific nuclear protein kinase, CLK1: CDC2-like kinase 1, CK1: casein kinase 1, GSK3: glycogen synthase kinase 3, ND: not determined.

a Values are a mean of $n \geq 3$ independent experiments.

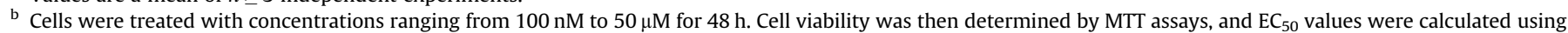
Graphpad PRISM 7 software $(n=3$ in triplicate; data are the mean $\pm S E M)$.

\subsection{Chemistry}

\subsubsection{Ethyl 3-oxo-3,4-dihydroquinoxaline-2-carboxylate (2)}

A mixture of $o$-phenylenediamine $(708 \mathrm{mg}, 6.55 \mathrm{mmol})$, diethyl 2-oxomalonate $(1.14 \mathrm{~g}, \quad 6.55 \mathrm{mmol})$ and citric acid (41 $\mathrm{mg}$, $0.20 \mathrm{mmol})$ in ethanol $(13 \mathrm{~mL})$ was stirred magnetically at room temperature for $10 \mathrm{~min}$. Ethanol was then evaporated under reduced pressure, and the residue was stirred with crushed ice for $5 \mathrm{~min}$, filtered and dried under vacuum to give compound $2(1.13 \mathrm{~g}$, $79 \%)$ as a beige solid.

Mp $168.8^{\circ} \mathrm{C} .{ }^{1} \mathrm{H}$ NMR (300 MHz, $\left.\mathrm{CDCl}_{3}\right) \delta 12.85$ (bs, $\left.1 \mathrm{H}, \mathrm{NH}\right), 7.97$ $(\mathrm{dd}, 1 \mathrm{H}, J=8.1,1.2 \mathrm{~Hz}$ ), 7.64 (dd, $1 \mathrm{H}, J=8.1,7.2,1.2 \mathrm{~Hz}), 7.48$ (dd, $1 \mathrm{H}$, $J=8.1,1.2 \mathrm{~Hz}), 7.42(\mathrm{dd}, 1 \mathrm{H}, J=8.1,7.2,1.2 \mathrm{~Hz}), 4.56(\mathrm{q}, 2 \mathrm{H}, J=7.2 \mathrm{~Hz}$, $\left.\mathrm{CH}_{2}\right), 1.49\left(\mathrm{t}, 3 \mathrm{H}, \mathrm{J}=7.2 \mathrm{~Hz}, \mathrm{CH}_{3}\right) \cdot{ }^{13} \mathrm{C} \mathrm{NMR}\left(75 \mathrm{MHz}, \mathrm{CDCl}_{3}\right) \delta 163.4$, $154.6,148.4,132.8,132.2,132.1,130.2,125.0,116.5,62.6,14.2$.

\subsubsection{Ethyl 3-chloroquinoxaline-2-carboxylate (3)}

Into a dry three-neck round bottom flask was introduced compound 2 (218 $\mathrm{mg}, 1.00 \mathrm{mmol}$ ) in phosphorous oxychloride $(2 \mathrm{~mL})$ at ice bath temperature. Dimethylformamide $(0.1 \mathrm{~mL})$ was then added at $0{ }^{\circ} \mathrm{C}$ and the reaction mixture was refluxed for $30 \mathrm{~min}$. After cooling, the resulting mixture was diluted with ethyl acetate and washed with a $10 \%$ sodium hydroxide solution $(2 \times 5 \mathrm{~mL})$, and brine $(2 \times 10 \mathrm{~mL})$. The combined organic layers were dried over $\mathrm{MgSO}_{4}$, filtered, and evaporated under reduced pressure to obtain derivative 3 ( $237 \mathrm{mg}, 100 \%$ ) as a beige solid.

Mp 46.4 ${ }^{\circ} \mathrm{C} .{ }^{1} \mathrm{H}$ NMR $\left(300 \mathrm{MHz}, \mathrm{CDCl}_{3}\right) \delta 8.18(\mathrm{~m}, 1 \mathrm{H}), 8.07(\mathrm{~m}$, $1 \mathrm{H}), 7.92-7.81(\mathrm{~m}, 2 \mathrm{H}), 4.58\left(\mathrm{q}, 2 \mathrm{H}, J=7.2 \mathrm{~Hz}, \mathrm{CH}_{2}\right), 1.49(\mathrm{t}, 3 \mathrm{H}$, $\left.J=7.2 \mathrm{~Hz}, \mathrm{CH}_{3}\right) .{ }^{13} \mathrm{C}$ NMR $\left(75 \mathrm{MHz}, \mathrm{CDCl}_{3}\right) \delta 163.9,144.7,143.9$, 142.2, 139.7, 132.6, 131.0, 129.6, 128.3, 63.0, 14.1.

\subsubsection{Ethyl 3-((3-hydroxyphenyl)amino)quinoxaline-2-carboxylate} (4a)

Method A: a solution of compound $3(1.11 \mathrm{~g}, 4.70 \mathrm{mmol}), 3-$ aminophenol (622 $\mathrm{mg}, 5.70 \mathrm{mmol}$ ) and $p$-TSA, as a catalyst, in absolute ethanol $(40 \mathrm{~mL})$ was refluxed for $110 \mathrm{~h}$. Ethanol was then evaporated under reduced pressure, and the resulting residue was purified by silica column chromatography using cyclohexane with ethyl acetate gradient $(0-50 \%)$ as eluent to give the desired compound $4 \mathbf{a}(1.0 \mathrm{~g}, 69 \%)$ as a red powder.

Mp 233. ${ }^{\circ} \mathrm{C} .{ }^{1} \mathrm{H}$ NMR (300 MHz, DMSO-d6) $\delta 10.08$ (bs, $1 \mathrm{H}, \mathrm{NH}$ ), 9.52 (bs, $1 \mathrm{H}, \mathrm{OH}), 7.99$ (dd, $1 \mathrm{H}, J=8.4,0.6 \mathrm{~Hz}), 7.85-7.75(\mathrm{~m}, 2 \mathrm{H})$, 7.61-7.54 (m, 2H), 7.24-7.14 (m, 2H), $6.51(\mathrm{dd}, 1 \mathrm{H}, J=7.4,2.4$, $1.5 \mathrm{~Hz}$ ), 4.48 (q, $\left.2 \mathrm{H}, J=7.2 \mathrm{~Hz}, \mathrm{CH}_{2}\right), 1.41\left(\mathrm{t}, 3 \mathrm{H}, J=7.2 \mathrm{~Hz}, \mathrm{CH}_{3}\right) .{ }^{13} \mathrm{C}$ NMR (75 MHz, DMSO-d6) $\delta$ 166.1, 158.3, 148.8, 142.4, 140.7, 136.0, 133.4, 132.7, $130.0(2 \times C), 126.7,126.6,111.1,110.6,107.3,62.8,14.5$.

\subsubsection{Ethyl 3-((3-aminophenyl)amino)quinoxaline-2-carboxylate (4b)}

The title compound was synthesized according to the general method A from compound 3 (330 mg, $1.40 \mathrm{mmol}$ ) and tert-butyl (3aminophenyl)carbamate $(312 \mathrm{mg}, 1.50 \mathrm{mmol}$ ) in absolute ethanol $(10 \mathrm{~mL})$. The reaction mixture was refluxed for $110 \mathrm{~h}$. Compound $\mathbf{4 b}$ was obtained (139 $\mathrm{mg}, 32 \%$ ) as a red powder.

Mp 207.7 ${ }^{\circ} \mathrm{C} .{ }^{1} \mathrm{H}$ NMR (300 MHz, DMSO-d6) $\delta 10.00$ (bs, $\left.1 \mathrm{H}, \mathrm{NH}\right)$, $7.97(\mathrm{~d}, 1 \mathrm{H}, J=8.1 \mathrm{~Hz}), 7.81-7.77(\mathrm{~m}, 2 \mathrm{H}), 7.58-7.51(\mathrm{~m}, 1 \mathrm{H}), 7.26$ (bs, 1H), 7.09-6.97 (m, 2H), $6.32(\mathrm{~m}, 1 \mathrm{H}), 5.17$ (bs, $\left.2 \mathrm{H}, \mathrm{NH}_{2}\right), 4.48$ (q, $\left.2 \mathrm{H}, J=7.2 \mathrm{~Hz}, \mathrm{CH}_{2}\right), 1.41$ (t, $\left.3 \mathrm{H}, J=7.2 \mathrm{~Hz}, \mathrm{CH}_{3}\right) .{ }^{13} \mathrm{C} \mathrm{NMR}(75 \mathrm{MHz}$, DMSO-d6) $\delta$ 166.1, 149.7, 149.0, 142.6, 140.3, 135.9, 133.3, 132.5, 130.0, 129.7, 126.7, 126.4, 109.7, 108.2, 105.8, 62.8, 14.5.

\subsubsection{Ethyl 3-((3-morpholinophenyl)amino)quinoxaline-2- carboxylate $(\mathbf{4 c})$}

The title compound was synthesized according to the general method A from compound 3 (361 $\mathrm{mg}, 1.53 \mathrm{mmol}$ ) and 3morpholinoaniline $(299 \mathrm{mg}, 1.68 \mathrm{mmol}$ ) in absolute ethanol $(10 \mathrm{~mL})$. The reaction mixture was refluxed for $110 \mathrm{~h}$. Compound $\mathbf{4 c}$ was obtained (439 $\mathrm{mg}, 76 \%$ ) as an orange powder.

Mp 170.6 ${ }^{\circ} \mathrm{C} .{ }^{1} \mathrm{H}$ NMR (300 MHz, DMSO-d6) $\delta 10.09$ (bs, $\left.1 \mathrm{H}, \mathrm{NH}\right)$, $7.98(\mathrm{~d}, 1 \mathrm{H}, J=8.1 \mathrm{~Hz}), 7.87-7.70(\mathrm{~m}, 2 \mathrm{H}), 7.67-7.47(\mathrm{~m}, 2 \mathrm{H}), 7.34(\mathrm{~d}$, $1 \mathrm{H}, J=7.8 \mathrm{~Hz}), 7.24(\mathrm{t}, 1 \mathrm{H}, J=8.1 \mathrm{~Hz}), 6.71(\mathrm{~d}, 1 \mathrm{H}, J=7.8 \mathrm{~Hz}), 4.48(\mathrm{q}$, $\left.2 \mathrm{H}, J=6.9 \mathrm{~Hz}, \mathrm{CH}_{2}\right), 3.77\left(\mathrm{~m}, 4 \mathrm{H}, 2 \times \mathrm{CH}_{2} \mathrm{O}\right.$ morpholine $), 3.16(\mathrm{~m}$, $4 \mathrm{H}, 2 \times \mathrm{CH}_{2} \mathrm{~N}$, morpholine), $1.41\left(\mathrm{t}, 3 \mathrm{H}, \mathrm{J}=6.9 \mathrm{~Hz}, \mathrm{CH}_{3}\right) .{ }^{13} \mathrm{C}$ NMR (75 MHz, DMSO-d6) $\delta$ 166.0, 152.2, 148.9, 142.4, 140.4, 136.0, 133.5, 132.6, 130.0, 129.8, $126.7(2 \times \mathrm{C}), 111.5,110.7,107.1,66.6(2 \times \mathrm{C}), 62.8$, $48.9(2 \times C), 14.5$. 
5.2.6. Ethyl 3-((1H-indol-5-yl)amino)quinoxaline-2-carboxylate (4d)

The title compound was synthesized according to the general method A from compound $3(237 \mathrm{mg}, 1.00 \mathrm{mmol})$ and 5aminoindole (397 $\mathrm{mg}, 3.00 \mathrm{mmol})$ in absolute ethanol $(10 \mathrm{~mL})$. The reaction mixture was refluxed for $36 \mathrm{~h}$. Compound $\mathbf{4 d}$ was obtained $(245 \mathrm{mg}, 73 \%)$ as a red powder.

Mp $204.7^{\circ} \mathrm{C} .{ }^{1} \mathrm{H}$ NMR (300 MHz, $\left.\mathrm{CDCl}_{3}\right) \delta 10.24$ (bs, $\left.1 \mathrm{H}, \mathrm{NH}\right), 8.80$ (bs, $1 \mathrm{H}, \mathrm{NH}$ ), 8.26 (s, $1 \mathrm{H}$, indolyl), 8.00 (dd, $1 \mathrm{H}, J=8.4,0.9 \mathrm{~Hz}$ ), 7.77 (dd, $1 \mathrm{H}, J=8.4,0.9 \mathrm{~Hz}$ ), 7.66 (dd, $1 \mathrm{H}, J=8.4,6.9,0.9 \mathrm{~Hz}$ ), 7.50-7.38 $(\mathrm{m}, 3 \mathrm{H}), 7.23(\mathrm{t}, 1 \mathrm{H}, J=2.4 \mathrm{~Hz}$, indolyl $), 6.56(\mathrm{bs}, 1 \mathrm{H}$, indolyl), 4.60 (q, $\left.2 \mathrm{H}, J=7.2 \mathrm{~Hz}, \mathrm{CH}_{2}\right), 1.53\left(\mathrm{t}, 3 \mathrm{H}, J=7.2 \mathrm{~Hz}, \mathrm{CH}_{3}\right) .{ }^{13} \mathrm{C} \mathrm{NMR}(75 \mathrm{MHz}$, $\left.\mathrm{CDCl}_{3}\right) \delta 166.4,150.0,143.6,136.2,133.0,132.8,131.5,130.6,130.1$, $128.1,126.6,125.4,125.1,117.2,112.8,111.2,102.6,62.9,14.3$.

\subsubsection{Ethyl 3-((4-hydroxyphenyl)amino)quinoxaline-2-carboxylate}

(4e)

The title compound was synthesized according to the general method A from compound 3 (302 $\mathrm{mg}, 1.28 \mathrm{mmol}$ ) and 4aminophenol (418 mg, $3.83 \mathrm{mmol}$ ) in absolute ethanol $(16 \mathrm{~mL})$. The reaction mixture was refluxed for $20 \mathrm{~h}$. Compound $\mathbf{4 e}$ was obtained (155 $\mathrm{mg}, 39 \%$ ) as a red powder.

Mp $231.8^{\circ} \mathrm{C} .{ }^{1} \mathrm{H}$ NMR (300 MHz, DMSO-d6) $\delta 9.84$ (bs, 1H, NH), 9.30 (bs, $1 \mathrm{H}, \mathrm{OH}), 7.94(\mathrm{~d}, 1 \mathrm{H}, J=8.1 \mathrm{~Hz}), 7.80-7.60(\mathrm{~m}, 4 \mathrm{H}), 7.51(\mathrm{~m}$, $1 \mathrm{H}), 6.80(\mathrm{~d}, 2 \mathrm{H}, J=8.7 \mathrm{~Hz}), 4.47\left(\mathrm{q}, 2 \mathrm{H}, J=7.2 \mathrm{~Hz}, \mathrm{CH}_{2}\right), 1.41(\mathrm{t}, 3 \mathrm{H}$, $\left.J=7.2 \mathrm{~Hz}, \mathrm{CH}_{3}\right) .{ }^{13} \mathrm{C}$ NMR (75 MHz, DMSO-d6) $\delta 166.0,154.0,149.2$, $142.8,135.9,133.3,132.5,131.0,130.0,126.5,126.1,122.7(2 \times \mathrm{C})$, $115.8(2 \times \mathrm{C}), 62.7,14.5$.

\subsubsection{Ethyl 3-((4-((tert-butoxycarbonyl)amino)phenyl)amino) quinoxaline-2-carboxylate (4f)}

The title compound was synthesized according to the general method A from compound 3 (95 mg, $0.40 \mathrm{mmol}$ ), tert-butyl (4aminophenyl)carbamate ( $250 \mathrm{mg}, 1.20 \mathrm{mmol}$ ) in absolute ethanol $(6.5 \mathrm{~mL})$. The reaction mixture was refluxed for $64 \mathrm{~h}$ in a sealed tube. After purification by silica column chromatography using $\mathrm{CH}_{2} \mathrm{Cl}_{2}$ with $\mathrm{MeOH}$ gradient $(0-2 \%)$ as eluent, compound $\mathbf{4 f}$ was obtained (152 $\mathrm{mg}, 93 \%)$ as an orange powder.

Mp 198.1 ${ }^{\circ} \mathrm{C} .{ }^{1} \mathrm{H}$ NMR (300 MHz, DMSO-d6) $\delta 10.00$ (bs, 1H, NH), 9.35 (bs, $1 \mathrm{H}, \mathrm{NH}), 8.00-7.40(\mathrm{~m}, 8 \mathrm{H}), 4.48\left(\mathrm{q}, 2 \mathrm{H}, J=7.2 \mathrm{~Hz}, \mathrm{CH}_{2}\right)$, $1.49\left(\mathrm{~s}, 9 \mathrm{H}, 3 \times \mathrm{CH}_{3}\right), 1.41\left(\mathrm{t}, 3 \mathrm{H}, J=7.2 \mathrm{~Hz}, \mathrm{CH}_{3} \mathrm{CH}_{2} \mathrm{O}\right)$.

\subsubsection{Ethyl 3-((4-aminophenyl)amino)quinoxaline-2-carboxylate} (4g)

To a solution of compound $\mathbf{4 f}(37 \mathrm{mg}, 0.09 \mathrm{mmol})$ in $\mathrm{CH}_{2} \mathrm{Cl}_{2}$ $(5 \mathrm{~mL})$ was added dropwise trifluoroacetic acid ( $1 \mathrm{~mL}, 13.06 \mathrm{mmol}$ ). The mixture was stirred at room temperature for $6 \mathrm{~h}$. The resulting mixture was made alkaline with a saturated sodium carbonate solution and extracted with $\mathrm{CH}_{2} \mathrm{Cl}_{2}$. The combined organic layers were dried over $\mathrm{MgSO}_{4}$, filtered, and evaporated under reduced pressure to give the desired derivative $\mathbf{4 g}(27 \mathrm{mg}, 96 \%)$ as a red powder.

Mp 203.3 ${ }^{\circ} \mathrm{C} .{ }^{1} \mathrm{H}$ NMR $\left(300 \mathrm{MHz}, \mathrm{CDCl}_{3}\right) \delta 10.06$ (bs, $\left.1 \mathrm{H}, \mathrm{NH}\right), 8.01$ (dd, $1 \mathrm{H}, J=8.4,1.2 \mathrm{~Hz}$ ), 7.80-7.64 (m, 4H), 7.44 (dd, $1 \mathrm{H}, J=8.1,6.6$, $1.2 \mathrm{~Hz}), 6.77(\mathrm{~d}, 2 \mathrm{H}, J=8.7 \mathrm{~Hz}), 4.61\left(\mathrm{q}, 2 \mathrm{H}, J=7.2 \mathrm{~Hz}, \mathrm{CH}_{2}\right), 3.70(\mathrm{bs}$, $\left.2 \mathrm{H}, \mathrm{NH}_{2}\right), 1.55\left(\mathrm{t}, 3 \mathrm{H}, J=7.2 \mathrm{~Hz}, \mathrm{CH}_{3}\right) .{ }^{13} \mathrm{C} \mathrm{NMR}\left(75 \mathrm{MHz}, \mathrm{CDCl}_{3}\right)$ $\delta$ 166.4, 149.7, 143.6, 142.6, 136.2, 132.8, 130.6, 130.5, 130.2, 126.6, $125.4,122.4(2 \times C), 115.5(2 \times C), 62.9,14.3$.

\subsubsection{Ethyl 3-((4-(1-methylpiperidin-4-yl)phenyl)amino) quinoxaline-2-carboxylate (4h)}

The title compound was synthesized according to the general method A from compound 3 (45 mg, $0.19 \mathrm{mmol}$ ) and 4-(1methylpiperidin-4-yl)aniline $(40 \mathrm{mg}, \quad 0.21 \mathrm{mmol})$ in absolute ethanol $(1.5 \mathrm{~mL})$. The reaction mixture was refluxed for $112 \mathrm{~h}$. After purification by silica column chromatography using $\mathrm{CH}_{2} \mathrm{Cl}_{2}$ with $\mathrm{MeOH}$ gradient $(0-10 \%)$ as eluent, compound $\mathbf{4 h}$ was obtained (47 $\mathrm{mg}, 63 \%$ ) as an orange powder.

$\mathrm{Mp} 127^{\circ} \mathrm{C} .{ }^{1} \mathrm{H}$ NMR $\left(300 \mathrm{MHz}, \mathrm{CDCl}_{3}\right) \delta 10.28$ (bs, $\left.1 \mathrm{H}, \mathrm{NH}\right), 8.02$ (dd, $1 \mathrm{H}, J=8.1,1.2 \mathrm{~Hz}), 7.84$ (d, 2H, $J=8.4 \mathrm{~Hz}$ ), 7.77 (dd, $1 \mathrm{H}, J=8.1$, $1.2 \mathrm{~Hz}$ ), 7.69 (dd, $1 \mathrm{H}, J=8.1,6.6,1.2 \mathrm{~Hz}$ ), 7.47 (dd, $1 \mathrm{H}, J=8.1,6.6$, $1.2 \mathrm{~Hz}), 7.26(\mathrm{~d}, 2 \mathrm{H}, J=8.4 \mathrm{~Hz}), 4.59\left(\mathrm{q}, 2 \mathrm{H}, J=7.2 \mathrm{~Hz}, \mathrm{CH}_{2}\right), 3.30(\mathrm{~d}$, $2 \mathrm{H}, J=11.7 \mathrm{~Hz}), 2.70-2.45(\mathrm{~m}, 6 \mathrm{H}), 2.24-2.09(\mathrm{~m}, 2 \mathrm{H}), 1.96(\mathrm{~d}, 2 \mathrm{H}$ $J=11.7 \mathrm{~Hz}), 1.53\left(\mathrm{t}, 3 \mathrm{H}, J=7.2 \mathrm{~Hz}, \mathrm{CH}_{3}\right) .{ }^{13} \mathrm{C} \mathrm{NMR}\left(75 \mathrm{MHz}, \mathrm{CDCl}_{3}\right)$ $\delta$ 166.4, 149.3, 143.2, 139.4, 137.7, 136.4, 132.9, 130.5, 130.2, 127.3 $(2 \times C), 126.7,125.9,120.5(2 \times C), 63.0,55.7(2 \times C), 45.1,40.4,31.9$ $(2 \times \mathrm{C}), 14.3$.

\subsubsection{Ethyl 3-((2-hydroxyphenyl)amino)quinoxaline-2-} carboxylate $\mathbf{4 i})$

The title compound was synthesized according to the general method A from compound $3(239 \mathrm{mg}, 1.01 \mathrm{mmol})$ and 2aminophenol $(121 \mathrm{mg}, 1.11 \mathrm{mmol})$ in absolute ethanol $(10 \mathrm{~mL})$. The reaction mixture was refluxed for $110 \mathrm{~h}$. Compound $4 \mathbf{i}$ was obtained (156 mg, 50\%) as an orange powder.

Mp 166.6 ${ }^{\circ} \mathrm{C} .{ }^{1} \mathrm{H}$ NMR (300 MHz, DMSO-d6) $\delta 10.66$ (bs, $1 \mathrm{H}, \mathrm{NH}$ ), 10.16 (bs, $1 \mathrm{H}, \mathrm{OH}), 8.89(\mathrm{~m}, 1 \mathrm{H}), 7.99(\mathrm{~d}, 1 \mathrm{H}, J=8.1 \mathrm{~Hz}), 7.80(\mathrm{~m}, 2 \mathrm{H})$, $7.56(\mathrm{~m}, 1 \mathrm{H}), 7.00-6.82(\mathrm{~m}, 3 \mathrm{H}), 4.49\left(\mathrm{q}, 2 \mathrm{H}, J=7.2 \mathrm{~Hz}, \mathrm{CH}_{2}\right), 1.43(\mathrm{t}$, $\left.3 \mathrm{H}, J=7.2 \mathrm{~Hz}, \mathrm{CH}_{3}\right) .{ }^{13} \mathrm{C}$ NMR (75 MHz, DMSO-d6) $\delta 165.8,149.0$, 147.0, 142.6, 135.9, 133.5, 132.3, 130.1, 128.4, 126.6, 126.5, 123.1, 119.7, 119.6, 114.7, 62.7, 14.6.

\subsubsection{3-((3-Hydroxyphenyl)amino)quinoxaline-2-carboxylic acid}

\section{(1)}

Method B: to ester $\mathbf{4 a}(72 \mathrm{mg}, 0.23 \mathrm{mmol})$ in aqueous methanol $(80 \%, 10 \mathrm{~mL})$, was added potassium carbonate $(97 \mathrm{mg}, 0.70 \mathrm{mmol})$ and the reaction mixture was refluxed for $4 \mathrm{~h}$. After cooling, the solvent was removed under reduced pressure. Then, the residue was acidified with a saturated citric acid aqueous solution, and extracted with ethyl acetate. The combined organic layers were dried over $\mathrm{MgSO}_{4}$, filtered, and evaporated under reduced pressure to yield the acid $\mathbf{1}$ ( $33 \mathrm{mg}, 50 \%$ ) as a red powder.

Mp 199.6 ${ }^{\circ} \mathrm{C} .{ }^{1} \mathrm{H}$ NMR (300 MHz, DMSO-d6) $\delta 10.52$ (bs, $\left.1 \mathrm{H}, \mathrm{NH}\right)$, 9.51 (bs, $1 \mathrm{H}, \mathrm{OH}), 7.98(\mathrm{~d}, 1 \mathrm{H}, J=8.4 \mathrm{~Hz}), 7.84-7.76(\mathrm{~m}, 2 \mathrm{H})$, $7.60-7.54(\mathrm{~m}, 2 \mathrm{H}), 7.25-7.14(\mathrm{~m}, 2 \mathrm{H}), 6.50(\mathrm{~d}, 1 \mathrm{H}, J=7.8 \mathrm{~Hz}) .{ }^{13} \mathrm{C}$ NMR (75 MHz, DMSO-d6) $\delta$ 168.1, 158.3, 149.3, 142.5, 140.7, 136.0, 133.2, 132.9, 130.1, 130.0, 126.6, 126.5, 111.0, 110.5, 107.2. HRMS (ESI) $m / z:[\mathrm{M}+\mathrm{H}]^{+}$calcd for $\mathrm{C}_{15} \mathrm{H}_{12} \mathrm{~N}_{3} \mathrm{O}_{3}, 282.2742$; found, 282.0873 .

\subsubsection{Potassium 3-((3-aminophenyl)amino)quinoxaline-2-} carboxylate $(\mathbf{5 b})$

Method C: to ester $\mathbf{4 b}(82 \mathrm{mg}, 0.26 \mathrm{mmol})$ in aqueous methanol $(80 \%, 10 \mathrm{~mL})$ was added potassium carbonate $(37 \mathrm{mg}, 0.26 \mathrm{mmol})$, and the reaction mixture was refluxed for $4 \mathrm{~h}$. After cooling, the solvent was removed under reduced pressure, and freeze-dried to obtain compound $\mathbf{5 b}(84 \mathrm{mg}, 100 \%)$ as an orange powder.

$\mathrm{Mp}>375^{\circ} \mathrm{C} .{ }^{1} \mathrm{H}$ NMR $(300 \mathrm{MHz}$, DMSO-d6) $\delta 13.15$ (bs, $1 \mathrm{H}, \mathrm{NH})$, $7.82(\mathrm{dd}, 1 \mathrm{H}, J=8.4,1.5 \mathrm{~Hz}), 7.65(\mathrm{dd}, 1 \mathrm{H}, J=7.2,1.8 \mathrm{~Hz}), 7.58(\mathrm{td}, 1 \mathrm{H}$, $J=7.2,1.5 \mathrm{~Hz}), 7.38(\mathrm{dd}, 1 \mathrm{H}, J=8.4,7.2,1.8 \mathrm{~Hz}), 7.21(\mathrm{t}, 1 \mathrm{H}$, $J=1.8 \mathrm{~Hz}), 7.14(\mathrm{dd}, 1 \mathrm{H}, J=7.8,1.8 \mathrm{~Hz}), 6.97(\mathrm{t}, 1 \mathrm{H}, J=7.8 \mathrm{~Hz}), 6.22$ (dd, $1 \mathrm{H}, J=7.8,1.8 \mathrm{~Hz}$ ), 5.04 (bs, $\left.2 \mathrm{H}, \mathrm{NH}_{2}\right) .{ }^{13} \mathrm{C}$ NMR ( $75 \mathrm{MHz}$, DMSOd6) $\delta$ 166.7, 150.4, 149.6, 143.2, 141.6, 141.5, 136.6, 130.2, 129.5 $(2 \times \mathrm{C}), 125.9,124.4,108.5,107.5,104.9$. HRMS (ESI) $m / z:[\mathrm{M}+\mathrm{H}]^{+}$ calcd for $\mathrm{C}_{15} \mathrm{H}_{13} \mathrm{~N}_{4} \mathrm{O}_{2}, 281.2895$; found, 281.1032.

\subsubsection{3-((3-Morpholinophenyl)amino)quinoxaline-2-carboxylic acid $(5 \mathrm{c})$}

The title compound was synthesized according to the general method B from compound $4 \mathrm{c}(150 \mathrm{mg}, 0.40 \mathrm{mmol})$ and potassium carbonate $(218 \mathrm{mg}, 1.58 \mathrm{mmol})$ in aqueous methanol $(80 \%, 5 \mathrm{~mL})$. 
The reaction mixture was refluxed for $4 \mathrm{~h}$. Compound $\mathbf{5 c}$ was obtained (139 $\mathrm{mg}, 100 \%$ ) as an orange powder.

Mp 186.6 ${ }^{\circ} \mathrm{C} .{ }^{1} \mathrm{H}$ NMR (300 MHz, DMSO-d6) $\delta 10.49$ (bs, $1 \mathrm{H}, \mathrm{NH}$ ), $7.97(\mathrm{~m}, 1 \mathrm{H}), 7.82-7.74(\mathrm{~m}, 2 \mathrm{H}), 7.67(\mathrm{~s}, 1 \mathrm{H}), 7.56(\mathrm{dd}, 1 \mathrm{H}, J=8.4,6.3$, $2.1 \mathrm{~Hz}) 7.32(\mathrm{~d}, 1 \mathrm{H}, J=8.7 \mathrm{~Hz}), 7.23(\mathrm{t}, 1 \mathrm{H}, J=8.1 \mathrm{~Hz}), 6.69(\mathrm{dd}, 1 \mathrm{H}$, $J=8.1,1.5 \mathrm{~Hz}$ ), $3.77\left(\mathrm{~m}, 4 \mathrm{H}, 2 \times \mathrm{CH}_{2} \mathrm{O}\right.$ morpholine), $3.16(\mathrm{~m}, 4 \mathrm{H}$, $2 \times \mathrm{CH}_{2} \mathrm{~N}$, morpholine). ${ }^{13} \mathrm{C}$ NMR (75 MHz, DMSO-d6) $\delta 168.0,152.2$, $149.4,142.5,140.5,135.9,133.2,132.9,129.9,129.7,126.7,126.4$, $111.4,110.5,106.9,66.6(2 \times \mathrm{C}), 49.0(2 \times \mathrm{C})$. HRMS $(\mathrm{ESI}) \mathrm{m} / \mathrm{z}$ : $[\mathrm{M}+\mathrm{H}]^{+}$calcd for $\mathrm{C}_{19} \mathrm{H}_{19} \mathrm{~N}_{4} \mathrm{O}_{3}, 351.3793$; found, 351.1453 .

\subsubsection{3-((1H-indol-5-yl)amino)quinoxaline-2-carboxylic acid} (5d)

To ester $4 \mathbf{d}(81 \mathrm{mg}, 0.24 \mathrm{mmol})$ in ethanol $(5 \mathrm{~mL})$, was added a $10 \%$ sodium hydroxide solution $(2 \mathrm{~mL})$ and the reaction mixture was refluxed for $18 \mathrm{~h}$. Ethanol was then evaporated under reduced pressure, and the resulting residue was acidified to $\mathrm{pH} 2$ with a $15 \%$ citric acid solution and extracted with ethyl acetate. The organic layers were then washed with water and brine, dried over $\mathrm{MgSO}_{4}$, filtered, and evaporated under reduced pressure. The residue was finally purified by silica column chromatography using $\mathrm{CH}_{2} \mathrm{Cl}_{2}$ with MeOH gradient (0-20\%) as eluent to give the acid $\mathbf{5 d ~ ( 3 0 ~ m g , ~ 4 1 \% ) ~}$ as a red powder.

Mp $244.4{ }^{\circ} \mathrm{C} .{ }^{1} \mathrm{H}$ NMR (300 MHz, DMSO-d6) $\delta 12.45$ (bs, $1 \mathrm{H}, \mathrm{NH}$ ), 11.07 (bs, $1 \mathrm{H}, \mathrm{NH}), 8.70-6.80(\mathrm{~m}, 8 \mathrm{H}), 6.44$ (bs, $1 \mathrm{H}$, indolyl). ${ }^{13} \mathrm{C}$ NMR (75 MHz, DMSO-d6) $\delta$ 168.6, 151.4, 133.6, 133.1, 132.5, 129.9, $129.4,129.1(3 \times C), 127.1,126.9,125.7,116.6,112.9,111.6,102.5$. HRMS (ESI) $m / z$ : $[\mathrm{M}+\mathrm{H}]^{+}$calcd for $\mathrm{C}_{17} \mathrm{H}_{13} \mathrm{~N}_{4} \mathrm{O}_{2}, 305.3109$; found, 305.1034 .

\subsubsection{3-((4-Hydroxyphenyl)amino)quinoxaline-2-carboxylic acid} (5e)

The title compound was synthesized according to the general method B from compound $4 \mathbf{e}(90 \mathrm{mg}, 0.29 \mathrm{mmol})$ and potassium carbonate $(80 \mathrm{mg}, 0.58 \mathrm{mmol})$ in aqueous methanol $(80 \%, 10 \mathrm{~mL})$. The reaction mixture was refluxed for $4 \mathrm{~h}$. Compound $5 \mathrm{e}$ was obtained ( $79 \mathrm{mg}, 97 \%$ ) as an orange powder.

Mp 197.4 ${ }^{\circ} \mathrm{C} .{ }^{1} \mathrm{H}$ NMR (300 MHz, DMSO-d6) $\delta 10.22$ (bs, $1 \mathrm{H}, \mathrm{NH}$ ), 9.31 (bs, $1 \mathrm{H}, \mathrm{OH}), 7.93$ (dd, $1 \mathrm{H}, J=8.1,0.9 \mathrm{~Hz}), 7.78-7.63(\mathrm{~m}, 4 \mathrm{H})$, $7.51(\mathrm{dd}, 1 \mathrm{H}, J=8.1,6.6,1.5 \mathrm{~Hz}), 6.80(\mathrm{~d}, 2 \mathrm{H}, J=8.7 \mathrm{~Hz}) .{ }^{13} \mathrm{C} \mathrm{NMR}$ (75 MHz, DMSO-d6) $\delta$ 168.1, 153.9, 149.6, 142.9, 135.8, 133.2, 132.7, 131.1, 129.9, 126.4, 125.9, $122.5(2 \times \mathrm{C}), 115.8(2 \times \mathrm{C})$. HRMS $(\mathrm{ESI}) \mathrm{m} /$ $z:[\mathrm{M}+\mathrm{H}]^{+}$calcd for $\mathrm{C}_{15} \mathrm{H}_{12} \mathrm{~N}_{3} \mathrm{O}_{3}, 282.2742$; found, 282.0873 .

\subsubsection{Potassium 3-((4-aminophenyl)amino)quinoxaline-2- carboxylate $(\mathbf{5 g})$}

The title compound was synthesized according to the general method $\mathrm{C}$ from compound $\mathbf{4 g}(16 \mathrm{mg}, 0.05 \mathrm{mmol})$ and potassium carbonate $(7 \mathrm{mg}, 0.05 \mathrm{mmol})$ in aqueous methanol $(80 \%, 5 \mathrm{~mL})$. The reaction mixture was refluxed for $4 \mathrm{~h}$. Compound $\mathbf{5 g}$ was obtained (14 $\mathrm{mg}, 87 \%$ ) as a red powder.

$\mathrm{Mp}>375^{\circ} \mathrm{C} .{ }^{1} \mathrm{H}$ NMR $(300 \mathrm{MHz}$, DMSO-d6) $\delta 12.80$ (bs, $1 \mathrm{H}, \mathrm{NH})$, $7.84(\mathrm{~d}, 1 \mathrm{H}, J=7.8 \mathrm{~Hz}), 7.60-7.50(\mathrm{~m}, 4 \mathrm{H}), 7.32(\mathrm{~m}, 1 \mathrm{H}), 6.60(\mathrm{~d}, 2 \mathrm{H}$, $J=8.4 \mathrm{~Hz}), 4.84$ (bs, $\left.2 \mathrm{H} \mathrm{NH}_{2}\right) .{ }^{13} \mathrm{C}$ NMR (75 MHz, DMSO-d6) $\delta 167.0$, $150.4,144.2,143.0,142.0,136.4,130.4,130.2,129.5,125.6,123.8$, $120.9(2 \times \mathrm{C}), 114.7(2 \times \mathrm{C})$. HRMS $(\mathrm{ESI}) \mathrm{m} / \mathrm{z}:[\mathrm{M}+\mathrm{H}]^{+}$calcd for $\mathrm{C}_{15} \mathrm{H}_{13} \mathrm{~N}_{4} \mathrm{O}_{2}$, 281.2895; found, 281.1032.

\subsubsection{3-((4-(1-Methylpiperidin-4-yl)phenyl)amino)quinoxaline- 2-carboxylic acid (5h)}

The title compound was synthesized according to the general method B from compound $\mathbf{4 h}(42 \mathrm{mg}, 0.11 \mathrm{mmol})$ and potassium carbonate $(45 \mathrm{mg}, 0.32 \mathrm{mmol}$ ) in aqueous methanol $(80 \%, 5 \mathrm{~mL})$. The reaction mixture was refluxed for $4 \mathrm{~h}$. Compound $\mathbf{5 h}$ was obtained (19 $\mathrm{mg}, 49 \%$ ) as a yellow powder.
Mp $321{ }^{\circ} \mathrm{C} .{ }^{1} \mathrm{H}$ NMR (300 MHz, DMSO-d6) $\delta 13.33$ (bs, 1H, NH), $8.00(\mathrm{~d}, 1 \mathrm{H}, J=7.8 \mathrm{~Hz}), 7.85(\mathrm{~d}, 2 \mathrm{H}, J=8.4 \mathrm{~Hz}), 7.68-7.58(\mathrm{~m}, 2 \mathrm{H})$, $7.54-7.31(\mathrm{~m}, 1 \mathrm{H}), 7.23$ (d, $2 \mathrm{H}, J=8.4 \mathrm{~Hz}), 2.86(\mathrm{~d}, 2 \mathrm{H}, J=11.4 \mathrm{~Hz})$, $2.46-2.36(\mathrm{~m}, 1 \mathrm{H}), 2.19\left(\mathrm{~s}, 3 \mathrm{H}, \mathrm{CH}_{3}\right), 1.95(\mathrm{td}, 2 \mathrm{H}, J=11.4,1.8 \mathrm{~Hz})$, $1.77-1.62(\mathrm{~m}, 4 \mathrm{H}) .{ }^{13} \mathrm{C}$ NMR (75 MHz, DMSO-d6) $\delta$ 166.9, 150.4, 142.1, 141.7, 140.1, 138.8, 136.4, 130.7, 129.8, 127.5 (2 $2 \times$ C), 125.9, 124.7, $119.3(2 \times C), 56.4(2 \times C), 46.7,41.2,33.7(2 \times C)$. HRMS (ESI) $\mathrm{m} / \mathrm{z}$ : an abundant fragment ion has been observed at $\mathrm{m} / \mathrm{z} 213.1022$ that have been attributed to the loss of the 1-methylpiperidin-4-yl) phenyl)amino moiety from the quinoxaline ring to form the ion $\left[\mathrm{C}_{9} \mathrm{H}_{6} \mathrm{~N}_{2} \mathrm{O}_{2}+\mathrm{K}\right]^{+}\left(\mathrm{m} / \mathrm{z}\right.$ calcd for $\mathrm{C}_{9} \mathrm{H}_{6} \mathrm{KN}_{2} \mathrm{O}_{2}, 213.2545$; found, 213.1022)

\subsubsection{3-((2-Hydroxyphenyl)amino)quinoxaline-2-carboxylic acid (5i)}

The title compound was synthesized according to the general method B from compound $\mathbf{4 i}(101 \mathrm{mg}, 0.33 \mathrm{mmol})$ and potassium carbonate ( $135 \mathrm{mg}, 0.98 \mathrm{mmol})$ in aqueous methanol $(80 \%, 10 \mathrm{~mL})$. The reaction mixture was refluxed for $4 \mathrm{~h}$. Compound $5 \mathbf{i}$ was obtained $(60 \mathrm{mg}, 65 \%$ ) as a red powder.

Mp $191.1^{\circ} \mathrm{C} .{ }^{1} \mathrm{H}$ NMR (300 MHz, DMSO-d6) $\delta 14.00$ (bs, $1 \mathrm{H}$, $\mathrm{COOH}), 10.84$ (bs, 1H, NH), 10.11 (bs, $1 \mathrm{H}, \mathrm{OH}), 8.88$ (m, 1H), 7.97 (d, $1 \mathrm{H}, J=8.1 \mathrm{~Hz}), 7.80(\mathrm{~m}, 2 \mathrm{H}), 7.56(\mathrm{~m}, 1 \mathrm{H}), 7.00-6.68(\mathrm{~m}, 3 \mathrm{H}) .{ }^{13} \mathrm{C}$ NMR (75 MHz, DMSO-d6) $\delta$ 167.1, 148.7, 146.5, 142.2, 135.3, 132.7, 132.4, 129.4, 127.9, 126.1, 125.8, 122.5, 119.2, 119.0, 114.2. HRMS (ESI) $m / z:[\mathrm{M}+\mathrm{H}]^{+}$calcd for $\mathrm{C}_{15} \mathrm{H}_{12} \mathrm{~N}_{3} \mathrm{O}_{3}, 282.2742$; found, 282.0871 .

\subsubsection{3-((3-Morpholinophenyl)amino)quinoxaline-2- carboxamide (6)}

To a solution of compound $\mathbf{5 c}(50 \mathrm{mg}, 0.14 \mathrm{mmol})$ and $\mathrm{N}$ methylmorpholine $(31 \mu \mathrm{L}, 0.29 \mathrm{mmol})$ in dichloromethane $(5 \mathrm{~mL})$ at $0{ }^{\circ} \mathrm{C}$, was added ethyl chloroformate $(21 \mu \mathrm{L}, 0.21 \mathrm{mmol})$. The reaction mixture was stirred magnetically at $0{ }^{\circ} \mathrm{C}$ for $1 \mathrm{~h}$, and a $28 \%$ solution of ammonium hydroxide $(5 \mathrm{~mL})$ was added. The reaction was stirred overnight at room temperature and extracted with dichloromethane. The organic layer was then washed with water and brine, dried over $\mathrm{MgSO}_{4}$, filtered, and evaporated under reduced pressure to give the carboxamide 6 (50 mg, 100\%) as a red powder.

Mp 203.9 ${ }^{\circ} \mathrm{C} .{ }^{1} \mathrm{H}$ NMR (300 MHz, DMSO-d6) $\delta 11.52$ (bs, $1 \mathrm{H}, \mathrm{NH}$ ), 8.75 (bs, $\left.1 \mathrm{H}, \mathrm{NH}_{2}\right), 8.26$ (bs, $\left.1 \mathrm{H}, \mathrm{NH}_{2}\right), 7.93$ (d, $1 \mathrm{H}, J=8.1 \mathrm{~Hz}$ ), 7.82-7.50 (m, 2H), 7.69 (bs, 1H), $7.56(\mathrm{~m}, 1 \mathrm{H}), 7.32-7.20(\mathrm{~m}, 2 \mathrm{H})$, $6.69(\mathrm{~d}, 1 \mathrm{H}, J=8.1 \mathrm{~Hz}), 3.78\left(\mathrm{~m}, 4 \mathrm{H}, 2 \times \mathrm{CH}_{2} \mathrm{O}\right.$ morpholine $), 3.17(\mathrm{~m}$, $4 \mathrm{H}, 2 \times \mathrm{CH}_{2} \mathrm{~N}$, morpholine). ${ }^{13} \mathrm{C}$ NMR (75 MHz, DMSO-d6) $\delta 168.5$, 152.2, 149.4, 142.7, 140.6, 135.3, 133.1, 132.8, 129.8, 129.6, 126.7, $126.3,111.1,110.3,106.6,66.6(2 \times \mathrm{C}), 49.0(2 \times \mathrm{C})$. HRMS $(\mathrm{ESI}) \mathrm{m} / \mathrm{z}$ : $[\mathrm{M}+\mathrm{H}]^{+}$calcd for $\mathrm{C}_{19} \mathrm{H}_{20} \mathrm{~N}_{5} \mathrm{O}_{2}, 350.3946$; found, 350.1613 .

\subsection{Molecular modeling}

Molecular modeling studies were performed using SYBYL-X 1.3 software [33] running on a Dell precision T3400 workstation. The three-dimensional structure of compound $\mathbf{1}$ (under its carboxylate form to imitate physiological conditions) was built from a standard fragments library and optimized using the Tripos force field [34] including the electrostatic term calculated from Gasteiger and Hückel atomic charges. Powell's method available in Maximin2 procedure was used for energy minimization until the gradient value was smaller than $0.001 \mathrm{kcal} /\left(\mathrm{mol}^{*} \AA\right)$. The crystal structure of Pim-1 in complex with AMP-PNP at $1.6 \AA$ resolution (PDB ID 3A99) [35] was used as template for docking. Water molecules were removed from the coordinates set since no information about conserved water molecules is known for this chemical series in Pim-1. Flexible docking of compound 1 into ATP-binding site was performed using GOLD software [36]. The most stable docking 
model was selected according to the best scored conformation predicted by the GoldScore scoring function. Finally, the complex was energy-minimized using Powell's method available in Maximin2 procedure with the Tripos force field and a dielectric constant of 4.0 , until the gradient value reached $0.1 \mathrm{kcal} / \mathrm{mol}$.A.

\subsection{Biology}

\subsubsection{Mammalian protein kinase assays}

Kinase enzymatic activities were assayed in 384-well plates using the ADP-Glo ${ }^{\mathrm{TM}}$ assay kit (Promega, Madison, $\mathrm{WI}$ ) according to the recommendations of the manufacturer. This assay is a luminescent ADP detection assay that provides a homogeneous and high-throughput screening method to measure kinase activity by quantifying the amount of ADP produced during a kinase reaction. Briefly, the reactions were carried out in a final volume of $5 \mu \mathrm{L}$ for $30 \mathrm{~min}$ at $30^{\circ} \mathrm{C}$ in ADP-Glo buffer and $10 \mu \mathrm{M}$ ATP $(40 \mathrm{mM}$ Tris $\mathrm{pH}$ $7.5,20 \mathrm{mM} \mathrm{MgCl} 2$ and $0.1 \mathrm{mg} / \mathrm{mL}$ of BSA). After that, $5 \mu \mathrm{L}$ of ADP$\mathrm{Glo}^{\mathrm{TM}}$ Kinase Reagent was added to stop the kinase reaction. After an incubation time of $50 \mathrm{~min}$ at room temperature (rt), $10 \mu \mathrm{L}$ of Kinase Detection Reagent was added for $1 \mathrm{~h}$ at $\mathrm{rt}$. The transmitted signal was measured using the Envision (PerkinElmer, Waltham, MA) microplate luminometer and expressed in Relative Light Unit (RLU). In order to determine the half maximal inhibitory concentration $\left(\mathrm{IC}_{50}\right)$, the assays were performed in triplicate in the absence or presence of increasing doses of the tested compounds. Kinase activities are expressed in \% of maximal activity, i.e. measured in the absence of inhibitor. Peptide substrates were obtained from Proteogenix (Schiltigheim, France).

The following kinases were analyzed during this study: HsPim-1 (human proto-oncogene, recombinant, expressed in bacteria) was assayed with $0.8 \mu \mathrm{g} / \mu \mathrm{L}$ of histone $\mathrm{H} 1$ (Sigma \#H5505) as substrate; RnDYRK1A-kd (Rattus norvegicus, amino acids 1 to 499 including the kinase domain, recombinant, expressed in bacteria, DNA vector kindly provided by Dr. W. Becker, Aachen, Germany) was assayed with $0.033 \mu \mathrm{g} / \mu \mathrm{L}$ of the following peptide: KKISGRLSPIMTEQ as substrate; HsCDK2/CyclinA (cyclin-dependent kinase-2, human, kindly provided by Dr. A. Echalier-Glazer, Leicester, UK) was assayed with $0.8 \mu \mathrm{g} / \mu \mathrm{L}$ of histone $\mathrm{H} 1$ as substrate; HsCDK9/CyclinT (human, recombinant, expressed by baculovirus in Sf9 insect cells) was assayed with $0.27 \mu \mathrm{g} / \mu \mathrm{L}$ of the following peptide: YSPTSPSYSPTSPSYSPTSPSKKKK, as substrate; HsHaspin-kd (human, kinase domain, amino acids 470 to 798 , recombinant, expressed in bacteria) was assayed with $0.007 \mu \mathrm{g} / \mu \mathrm{L}$ of Histone $\mathrm{H} 3$ (1-21) peptide (ARTKQTARKSTGGKAPRKQLA) as substrate; MmCLK1 (from Mus musculus, recombinant, expressed in bacteria) was assayed with $0.027 \mu \mathrm{g} / \mu \mathrm{L}$ of the following peptide: GRSRSRSRSRSR as substrate; $S s c C K 1 \delta / \varepsilon$ (casein kinase $1 \delta / \varepsilon$, porcine brain, native, affinity purified) was assayed with $0.022 \mu \mathrm{g} / \mu \mathrm{L}$ of the following peptide: RRKHAAIGSpAYSITA ("Sp" stands for phosphorylated serine) as CK1-specific substrate; SscGSK-3 $\alpha / \beta$ (glycogen synthase kinase-3, porcine brain, native, affinity purified) isoforms were assayed with $0.010 \mu \mathrm{g} / \mu \mathrm{L}$ of GS-1 peptide, a GSK-3-selective substrate (YRRAAVPPSPSLSRHSSPHQSpEDEEE). To validate the kinase assay, model inhibitors were used for each tested enzyme: Staurosporine from Streptomyces sp. (\#S5921, purity $\geq 95 \%$, Sigma-Aldrich) for SscCK $1 \delta / \varepsilon$; Indirubin-3'-oxime (\#I0404, purity $\geq 98 \%$, SigmaAldrich) for SscGSK-3 $\alpha / \beta$, HsPim-1, human Cyclin-dependent kinases, RnDYRK1A and MmCLK1; CHR-6494 (\#SML0648, purity $\geq 98 \%$, Sigma-Aldrich) for Haspin.

\subsubsection{Cell cultures and reagents}

KU812 cell lines were obtained from the American Type Culture Collection (ATCC) and maintained according to the supplier's recommendations. Cell lines were cultured in RPMI, with $10 \%$ fetal bovine serum, $1 \%$ glutamine, and $1 \%$ penicillin/streptomycin at $37^{\circ} \mathrm{C}$ and $5 \% \mathrm{CO}_{2}$.

\subsubsection{Cell proliferation assays}

Cell viability and proliferation were studied using a MTT cell proliferation assay. Briefly, $0.2 \times 10^{5}$ cells were incubated in $100 \mu \mathrm{L}$ of X-Vivo red phenol free medium (Lonza, Basel, Switzerland) in 96 well plates. In initial screening assays, cells were incubated with $10 \mu \mathrm{M}$ of each compound (quinoxalines stock solution at $50 \mathrm{mM}$ in DMSO) for 24,48 , and $72 \mathrm{~h}$. Imatinib mesylate (Selleckchem, stock solution at $10 \mathrm{mM}$ in DMSO) was used as reference. To determine the concentration-effect of the molecules, cells were treated with concentrations ranging from $100 \mathrm{nM}$ to $50 \mu \mathrm{M}$ for 24 or $48 \mathrm{~h}$. Cells were incubated with $10 \mu \mathrm{L}$ of MTT working solution $(5 \mathrm{~g} / \mathrm{L}$ of methylthiazolyldiphenyl-tetrazolium bromide) during $4 \mathrm{~h}$. Cells were then lysed overnight at $37{ }^{\circ} \mathrm{C}$ with $100 \mu \mathrm{L}$ of $10 \%$ SDS and $0.003 \% \mathrm{HCl}$. Optical density (OD) at $570 \mathrm{~nm}$ was measured using a spectrophotometer (Dynex, Chantilly, United States). Living cells were also counted with the trypan blue dye exclusion method. When a dose-dependent activity was observed, $\mathrm{EC}_{50}$ values were calculated using Graphpad PRISM 7 software ( $n=3$ in triplicate). Data were collected from at least three independent experiments and the values reported are means \pm standard errors of the mean.

\section{Acknowledgments}

This work was supported by a grant from the «Association pour le Développement de la Recherche et de l'Innovation dans le NORD PAS DE CALAIS » (ADRINORD) (to B. O.). The authors thank the «Plateforme Scientifique et Technique Analyses des Systèmes Biologiques » (PST-ASB), Tours (France), for NMR spectrometry and the « Fédération de Recherche »ICOA/CBM (FR2708) platform, for HRMS analyses. The authors also thank the Cancéropôle Grand Ouest (axis: Natural sea products in cancer treatment), GIS IBiSA (Infrastructures en Biologie Santé et Agronomie) and Biogenouest (Western France life science and environment core facility network) for supporting KISSf screening facility. The authors wish to thank the Région Centre Val de Loire for financial support.

\section{Appendix A. Supplementary data}

Supplementary data related to this article can be found at https://doi.org/10.1016/j.ejmech.2018.04.056.

\section{References}

[1] H.T. Cuypers, G. Selten, W. Quint, M. Zijlstra, E.R. Maandag, W. Boelens, P. Van Wezenbeek, C. Melief, A. Berns, Murine leukemia virus-induced T-cell lymphomagenesis: integration of proviruses in a distinct chromosomal region, Cell 37 (1984) 141-150.

[2] T. Mochizuki, C. Kitanaka, K. Noguchi, T. Muramatsu, A. Asai, Y.J. Kuchino Physical and functional interactions between Pim-1 kinase and Cdc25A phosphatase. Implications for the Pim-1-mediated activation of the c-Myc signaling pathway, J. Biol. Chem. 274 (1999) 18659-18666.

[3] M. Bachmann, C. Kosan, P.X. Xing, M. Montenarh, I. Hoffmann, T. Möröy, The oncogenic serine/threonine kinase Pim-1 directly phosphorylates and activates the G2/M specific phosphatase Cdc25C, Int, J. Biochem. Cell Biol. 38 (2006) 430-443.

[4] R. Amson, F. Sigaux, S. Przedborski, G. Flandrin, D. Givoland, A. Telerman, The human protooncogene product p33pim is expressed during fetal hematopoiesis and in diverse leukemias, Proc. Natl. Acad. Sci. U. S. A 86 (1989) 8857-8861.

[5] E.D. Hsi, S.H. Jung, R. Lai, J.L. Johnson, J.R. Cook, D. Jones, S. Devos, B.D. Cheson, L.E. Damon, J. Said, Ki67 and PIM1 expression predict outcome in mantle cell lymphoma treated with high dose therapy, stem cell transplantation and rituximab: a Cancer and Leukemia Group B 59909 correlative science study Leuk. Lymphoma 49 (2008) 2081-2090.

[6] S.J. Guo, X.P. Mao, J.X. Chen, B. Huang, C. Jin, Z.B. Xu, S.P. Qiu, Overexpression of Pim-1 in bladder cancer, J. Exp. Clin. Canc. Res. 29 (2010) 161.

[7] S.M. Dhanasekaran, T.R. Barrette, D. Ghosh, R. Shah, S. Varambally, K. Kurachi, 
K.J. Pienta, M.A. Rubin, A.M. Chinnaiyan, Delineation of prognostic biomarkers in prostate cancer, Nature 412 (2001) 822-826.

[8] F. Brasó-Maristany, S. Filosto, S. Catchpole, R. Marlow, J. Quist, E. FranceschDomenech, D.A. Plumb, L. Zakka, P. Gazinska, G. Liccardi, P. Meier, A. GrisOliver, M.C. Cheang, A. Perdrix-Rosell, M. Shafat, E. Noël, N. Patel, K. McEachern, M. Scaltriti, P. Castel, F. Noor, R. Buus, S. Mathew, J. Watkins, V. Serra, P. Marra, A. Grigoriadis, A.N. Tutt, PIM1 kinase regulates cell death, tumor growth and chemotherapy response in triple-negative breast cancer, Nat. Med. 22 (2016) 1303-1313.

[9] B. Yan, E.X. Yau, S. Samanta, C.W. Ong, K.J. Yong, L.K. Ng, B. Bhattacharya, K.H. Lim, R. Soong, K.G. Yeoh, N. Deng, P. Tan, Y.L. Lam, M. Salto-Tellez, Clinica and therapeutic relevance of PIM1 kinase in gastric cancer, Gastric Cancer 15 (2012) 188-197.

[10] Y. Tursynbay, J. Zhang, Z. Li, T. Tokay, Z. Zhumadilov, D. Wu, Y. Xie, Pim-1 kinase as cancer drug target: an update, Biomed, For. Rep. 4 (2016) 140-146.

[11] H. Mikkers, M. Nawijn, J. Allen, C. Brouwers, E. Verhoeven, J. Jonkers, A. Berns, Mice deficient for all PIM kinases display reduced body size and impaired responses to hematopoietic growth factors, Mol. Cell Biol. 24 (2004) 6104-6115.

[12] R.A. Darby, A. Unsworth, S. Knapp, I.D. Kerr, R. Callaghan, Overcoming ABCG2mediated drug resistance with imidazo-[1,2-b]-pyridazine-based Pim1 kinase inhibitors, Cancer Chemother, Pharmacology (Basel) 76 (2015) 853-864.

[13] Y. Xie, S. Bayakhmetov, PIM1 kinase as a promise of targeted therapy in prostate cancer stem cells, Mol. Clin. Oncol 4 (2016) 13-17.

[14] C. Liang, Y.Y. Li, Use of regulators and inhibitors of Pim-1, a serine/threonine kinase, for tumour therapy (review), Mol. Med. Rep. 9 (2014) 2051-2060.

[15] F. Anizon, A.A. Shtil, V.N. Danilenko, P. Moreau, Fighting tumor cell survival: advances in the design and evaluation of Pim inhibitors, Curr. Med. Chem. 17 (2010) 4114-4133.

[16] Y. Xu, B.G. Brenning, S.G. Kultgen, J.M. Foulks, A. Clifford, S. Lai, A. Chan, S. Merx, M.V. McCullar, S.B. Kanner, K.-K. Ho, Synthesis and biological evaluation of pyrazolo[1,5-a]pyrimidine compounds as potent and selective Pim-1 inhibitors, ACS Med. Chem. Lett. 6 (2015) 63-67.

[17] H.-B. Sun, X.-Y. Wang, G.-B. Li, L.-D. Zhang, J. Liu, L.-F. Zhao, Design, synthesis and biological evaluation of novel C3-functionalized oxindoles as potential Pim-1 kinase inhibitors, RSC Adv. 5 (2015) 29456-29466.

[18] C. Blanco-Aparicio, A. Carnero, Pim kinases in cancer: diagnostic, prognostic and treatment opportunities, Biochem. Pharmacol. 85 (2013) 629-643.

[19] A. Kumar, V. Mandiyan, Y. Suzuki, C. Zhang, J. Rice, J. Tsai, D.R. Artis, P. Ibrahim, R. Bremer, Crystal structures of proto-oncogene kinase Pim1: a target of aberrant somatic hypermutations in diffuse large cell lymphoma, J. Mol. Biol. 348 (2005) 183-193.

[20] B.T. Le, M. Kumarasiri, J.R. Adams, M. Yu, R. Milne, M.J. Sykes, S. Wang, Targeting Pim kinases for cancer treatment: opportunities and challenges, Future Med. Chem. 7 (2015) 35-53.

[21] L. Juen, M. Brachet-Botineau, C. Parmenon, J. Bourgeais, O. Hérault, F. Gouilleux, M.C. Viaud-Massuard, G. Prié, New inhibitor targeting signal transducer and activator of transcription 5 (STAT5) signaling in myeloid leukemias, J. Med. Chem. 60 (2017) 6119-6136.

[22] Z. Guo, A. Wang, W. Zhang, M. Levit, Q. Gao, C. Barberis, M. Tabart, J. Zhang, D. Hoffmann, D. Wiederschain, J. Rocnik, F. Sun, J. Murtie, C. Lengauer, S. Gross,
B. Zhang, H. Cheng, V. Patel, L. Schio, F. Adrian, M. Dorsch, C. Garcia-Echeverria, S.M. Huang, PIM inhibitors target CD25-positive AML cells through concomitant suppression of STAT5 activation and degradation of MYC oncogene, Blood 124 (2014) 1777-1789.

[23] L.S. Chen, S. Redkar, D. Bearss, W.G. Wierda, V. Gandhi, Pim kinase inhibitor, SGI-1776, induces apoptosis in chronic lymphocytic leukemia cells, Blood 114 (2009) 4150-4157.

[24] Y. Xiang, B. Hirth, G. Asmussen, H.P. Biemann, K.A. Bishop, A. Good, M. Fitzgerald, T. Gladysheva, A. Jain, K. Jancsics, J. Liu, M. Metz, A. Papoulis, R. Skerlj, J.D. Stepp, R.R. Wei, The discovery of novel benzofuran-2-carboxylic acids as potent Pim-1 inhibitors, Bioorg. Med. Chem. Lett 21 (2011) 3050-3056.

[25] J. Bogusz, K. Zrubek, K.P. Rembacz, P. Grudnik, P. Golik, M. Romanowska, B. Wladyka, G. Dubin, Crystal structure of PIM1 kinase in complex with smallmolecule inhibitor, Sci. Rep. 7 (2017), 13399-13399.

[26] R. Mahesh, A.K. Dhar, T. Sasank T. V. N. V. S. Thirunavukkarasu, T. Devadoss, Citric acid: an efficient and green catalyst for rapid one pot synthesis of quinoxaline derivatives at room temperature, Chin. Chem. Lett. 22 (2011) 389-392.

[27] R. Mahesh, T. Devadoss, A.K. Dhar, S.M. Venkatesh, S. Mundra, D.K. Pandey, S. Bhatt, A.K. Jindal, Ligand-based design, synthesis, and pharmacological evaluation of 3-methoxyquinoxalin-2-carboxamides as structurally novel serotonin type-3 receptor antagonists, Arch. Pharm. Chem. Life Sci. 345 (2012) 687-694.

[28] A. Kumar, K. Srivastava, S.R. Kumar, M.I. Siddiqi, S.K. Puri, J.K. Sexana, P.M. Chauhan, 4-Anilinoquinoline triazines: a novel class of hybrid antimalarial agents, Eur. J. Med. Chem. 46 (2011) 676-690.

[29] H. Zegzouti, M. Zdanovskaia, K. Hsiao, S.A. Goueli, ADP-Glo: a Bioluminescent and homogeneous ADP monitoring assay for kinases, Assay Drug Dev. Technol. 7 (2009) 560-572.

[30] P. Lassalas, B. Gay, C. Lasfargeas, M.J. James, V. Tran, K.G. Vijayendran, K.R. Brunden, M.C. Kozlowski, C.J. Thomas, A.B. Smith III, D.M. Huryn, C. Ballatore, Structure property relationships of carboxylic acid isosteres, J. Med. Chem. 59 (2016) 3183-3203.

[31] F. Jahani, M. Tajbakhsh, H. Golchoubian, S. Khaksar, Guanidine hydrochloride as an organocatalyst for N-Boc protection of amino groups, Tetrahedron Lett. 52 (2011) 1260-1264.

[32] R. Wurz, A. Tasker, S. Tadesse, L.H. Pettus, T.T. Nguyen, F.-T. Hong B.J. Herberich, E. Harrington, J.J. Chen, J. Brown, Substituted 7-oxo-pyrido[2,3d] pyrimidines and their use for the treatment of EGFR/ErbB2 related disorders, From PCT Int. Appl (2014) 108-109. W02014134308.

[33] SYBYL-X 1.3, Tripos Associates, Inc, South Hanley Road, St. Louis, MO 63144, U.S.A, 1699.

[34] M. Clarck, R.D. Cramer III, N. Van Opdenbosch, Validation of the general purpose tripos 5.2 force field, J. Comput. Chem. 10 (1989) 982-1012.

[35] D. Morishita, M. Takami, S. Yoshikawa, R. Katayama, S. Sato, N. KukimotoNiino, T. Umehara, M. Shirouzu, K. Sekimizu, S. Yokoyama, N. Fujita, Cellpermeable carboxyl-terminal p27 ${ }^{\text {Kip } 1}$ peptide exhibits anti-tumor activity by inhibiting Pim-1 kinase, J. Biol. Chem. 286 (2011) 2681-2688.

[36] G. Jones, P. Willet, R.C. Glen, Development and validation of a genetic algorithm for flexible docking, J. Mol. Biol. 267 (1997) 727-748. 